\title{
Long-term results of total knee arthroplasty with single-radius versus multi-radius posterior-stabilized prostheses
}

\author{
Zhenyu Luo(D, Zeyu Luo, Haoyang Wang, Qiang Xiao, Fuxing Pei and Zongke Zhou* (D)
}

\begin{abstract}
Background: Single-radius (SR) prostheses and multi-radius (MR) prostheses have different theoretical advantages; however, there has been a paucity of evaluations comparing the two. This study was designed to compare the 10-year clinical, radiological, and survival outcomes of SR and MR posterior-stabilized prostheses in total knee arthroplasty (TKA).

Methods: In this retrospective cohort study, 220 consecutive patients undergoing TKA between October 2006 and October 2007 were divided into the SR group (106 patients, Stryker Scorpio NRG) and the MR group (114 patients, DePuy Sigma PFC), with a minimum follow-up of 10 years. Clinical, functional, and radiological outcomes, as well as satisfaction rates and survival results, were evaluated.

Results: Hospital for Special Surgery and Short Form-12 health survey scores were all significantly improved in both groups at the final follow-up $(P<0.05)$, but the groups did not differ. The SR group had significantly less anterior knee pain (AKP) and painless crepitation $(P<0.05)$. Radiological results in terms of radiolucent lines and component position angle showed no differences between groups. The Kaplan-Meier survival curve estimates at 10 years were not significantly different between the groups $(P=0.4172)$.

Conclusion: Both SR and MR posterior-stabilized prostheses can lead to satisfactory outcomes. The SR prosthesis design gave less anterior knee pain than did the MR prostheses. Two prostheses showed no differences in terms of clinical scales, radiological results, satisfaction rates, and survival results at a long-term follow-up. More accurate measurements are required.
\end{abstract}

Keywords: Total knee arthroplasty, Single-radius, Multi-radius, Long-term results, Anterior knee pain

\section{Background}

Total knee arthroplasty (TKA) excellent operation rate has reached $90 \%$ [1], and the revision rate has been less than $4 \%[2]$. However, the satisfaction rate of patients with TKA has only been $75-80 \%$, and $10 \%$ of patients still experience anterior knee pain (AKP) or patellofemoral complications [3].

The first theory of the knee rotation center was proposed by Frankel et al. [4] in 1971. They described knee flexion occurring around a varying transverse axis, with the instantaneous rotation center of the femoral posterior

\footnotetext{
* Correspondence: zhouzongke@scu.edu.cn

Department of Orthopedics, West China Hospital/West China School of

Medicine, Sichuan University, 37\# Wuhou Guoxue road, Chengdu 610041

People's Republic of China
}

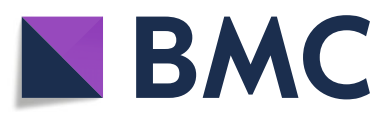

(c) The Author(s). 2019 Open Access This article is distributed under the terms of the Creative Commons Attribution 4.0 International License (http://creativecommons.org/licenses/by/4.0/), which permits unrestricted use, distribution, and reproduction in any medium, provided you give appropriate credit to the original author(s) and the source, provide a link to the Creative Commons license, and indicate if changes were made. The Creative Commons Public Domain Dedication waiver (http://creativecommons.org/publicdomain/zero/1.0/) applies to the data made available in this article, unless otherwise stated.

condyle forming a "J curve" $[4,5]$. The multi-radius (MR) femoral prosthesis was theoretically designed, and the trans-epicondylar axis (TEA) which was perpendicular to the mechanical alignment (MA) of the lower extremity was widely used as a skeletal marker for the axis of flexion and rotation [6]. The multi-radius femoral prosthesis was designed based on this theory and was widely utilized since the 1980s. For example, in the sigma PFC system, the femoral implant had three segment progressive radius at the sagittal plane, and in the recent MR prostheses, the Attune system has been a continuously progressive radius like a spiral cord which may provide a more fluent flexion movement [7]. 
However, in 1993, Hollister et al. [8] demonstrated that the actual flexion-extension axis (FEA) of the knee sometimes did not coincide with the epicondylar axis and had a more distal and posterior axis. The locus of the rotation center between 10 and $120^{\circ}$ can be regarded as a single spherical radius $[8,9]$. The single-radius (SR) femoral prosthesis was designed according to this theory. For example, the first SR prosthesis, the Scorpio single-radius TKA (Stryker, Mahwah, NJ), was produced in 1996 [10]. The second stage was the Scorpio NRG system, and it is now the latest stage with high-flexion system in the Triathlon TKA prosthesis $[11,12]$.

The SR prosthesis provided a more distal FEA to have a longer extensor moment arm [13], maintaining stabilization during the middle segment range of motion (ROM), thereby reducing the paradoxical anterior femoral movement [14], alleviating anterior knee pain (AKP), and providing a better patellofemoral trajectory [11]. Although SR prosthesis designs had theoretical advantages, there has been lack of sufficient quality studies. Most of these studies included no more than 50 patients in each group or followed up for no longer than 3 years [15-18], as shown in Table 5. Finally, some studies suffered from methodological shortcomings.

The aim of this retrospective cohort study was to compare the clinical results between $\mathrm{SR}$ and $\mathrm{MR}$ posterior-stabilized (PS) prostheses. We hypothesized that both two prostheses can provide satisfactory results and the SR prostheses provide similar functional, radiological, and survival results to the MR prostheses in TKA.

\section{Methods}

The retrospective cohort study was approved by the Institutional Review Board of West China Hospital, Sichuan University (ID number: 2012-268). The work was registered in the Chinese Clinical Trial Registry (ID number: ChiCTR1800016129). Informed consent was obtained from all patients and their relatives. Information about deceased participants was collected from their relatives.

This retrospective cohort study included a consecutive series of 327 patients who underwent TKA by one surgery group in West China Hospital between October 2006 and October 2007. We included patients who underwent primary unilateral TKA with SR (Stryker Scorpio NRG) or MR (DePuy sigma PFC) prostheses. The two prostheses were the most utilized prostheses during that time period. We declared no additional information about the implants to patients, so which implant to utilize was irrelevant to characteristics of the patients or severity of the symptoms and was only chosen by patients themselves for their own desires.

We excluded patients with revision TKA, patients diagnosed with inflammatory arthritis, patients who underwent bilateral TKA, patients who required cruciate-retain (CR) or any other types of SR or MR prosthesis, patients who required cones or augments for severe bone defects, and patients who could not give informed consent. Therefore, 65 patients were excluded from the study for any reasons (Fig. 1). At the final 10-year follow-up, 42 patients were lost to follow-up, underwent revision, or died (Table 3). Accordingly, the final follow-up was conducted

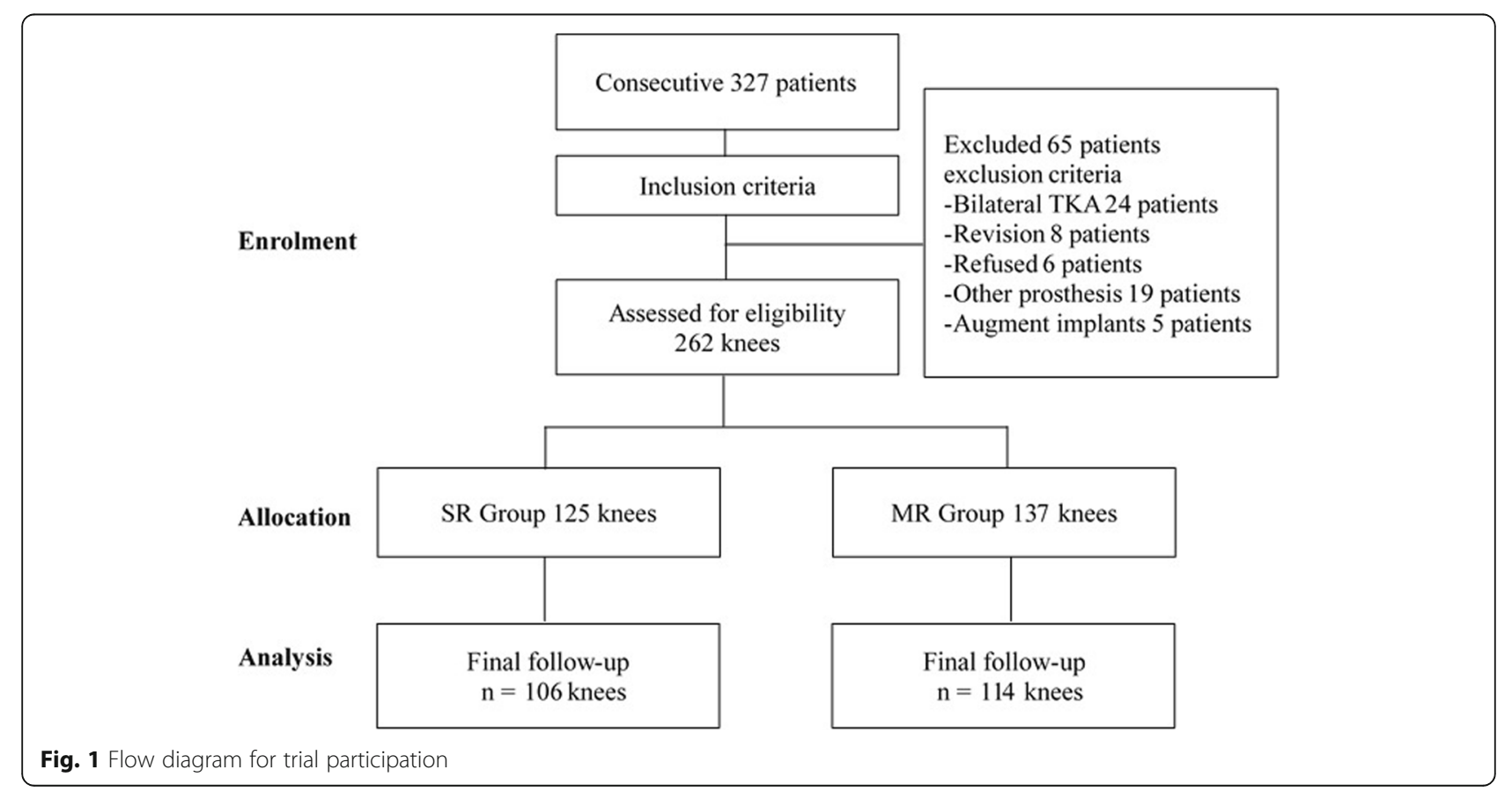


on 220 patients, among whom 106 received SR prostheses and 114 patients received MR prostheses. Baseline data from the two groups are listed in Table 1. There were no significant differences in terms of mean age, gender, BMI, or diagnoses of disease.

\section{Surgical technique}

One surgery team performed all operations. The SR prostheses were Scorpio NRG (Stryker Orthopedics, Mahwah, NJ, USA) and the SR prostheses were Sigma PFC (DePuy Orthopedics, IN, USA). All K-L (Kellgren-Lawrance) arthritis grades of the patients were in grade IV. A medial parapatellar approach was used. Distal femoral osteotomies were usually $6^{\circ}$ to the femur anatomic axis [19]. Femoral rotational alignment was performed according to the epicondylar axis, usually $3^{\circ}$ of external rotation from the posterior condylar line. Both groups removed the posterior cruciate ligaments (PCL). Femoral components were similar size (standard size) in both designs, and the cemented tibial baseplates were also similar in both designs. Patellar resurfacing was not utilized in either group. All components were cemented. All patients were given cephalosporin for $24 \mathrm{~h}$ to prevent infection. A plasma drainage tube was used for $24 \mathrm{~h}$. Continuous movement exercises were started postoperatively to recover quadriceps strength.

\section{Evaluation}

Patients were regularly followed up every 6 months after surgery. The evaluation was conducted using HSS (Hospital for Special Surgery) scores, KSS (Knee Society score, knee and function), WOMAC (The Western Ontario and McMaster Universities) score, SF-12 scale (the Short Form-12 health survey, physical and mental), and ROM (range of motion, flexion and extension). The VAS (visual analog scale) and incidence of anterior knee pain and

Table 1 Demographic data of patients at final follow-up

\begin{tabular}{llll}
\hline & SR group & MR group & $P$ value \\
\hline Patients & 106 & 114 & - \\
Gender (female/male) & $82 / 24$ & $78 / 36$ & 0.1369 \\
Age & $69.54 \pm 10.57$ & $68.98 \pm 10.23$ & 0.8418 \\
BMI & $23.38 \pm 2.58$ & $23.45 \pm 2.61$ & 0.6901 \\
Follow-up years & $10.72 \pm 1.78$ & $10.65 \pm 1.87$ & - \\
Diagnoses & & & \\
$\quad$ Primary osteoarthritis & $81(76.74 \%)$ & $85(78.31 \%)$ & 0.7496 \\
$\quad$ Rheumatic arthritis & $20(17.44 \%)$ & $23(15.66 \%)$ & 0.8069 \\
$\quad$ Posttraumatic arthritis & $2(2.33 \%)$ & $2(2.41 \%)$ & 0.3769 \\
$\quad$ Gouty arthritis & $3(3.49 \%)$ & $4(3.61 \%)$ & 0.2864 \\
\hline
\end{tabular}

Continuous data presented as the mean \pm std. Student's $t$ test was utilized. Discontinuous data presented as frequencies (percentages). Pearson's chisquared test or Fisher's exact test was utilized. $P<0.05$ indicates significant differences crepitation were recorded. The chair test was used to exam the stability of the prosthesis indirectly, allowing patients to stand from a chair without the assistance of both arms on the armrests [10]. The satisfaction rate was also evaluated.

Radiological evaluation was based on weight-bearing standing posterior-anterior, lateral view radiographs. Location of radiolucent lines was evaluated based on the Knee Society Roentgenographic Evaluation System [20]. The component positions were evaluated by four angles: the lateral distal femoral angle (LDFA, $\alpha$ angle), which is measured between the anatomical axis of the femur and a tangential line to the distal condyles of the femoral prosthesis; the medial proximal tibia angle (MDTA, $\beta$ angle), which lies between the anatomical axis of the tibia and a tangential line to the plateau of the tibial prosthesis; the flexion-extension femoral angle (FEFA, $\gamma$ angle), which is between a line through the midshaft of the femur and the neutral line of the femoral prosthesis; and the tibial slope angle (TSA, $\sigma$ angle), which is described by a line through the midshaft of the tibia and a tangential line to the tibial prosthesis [21] (Fig. 2c, d).

Kaplan-Meier survival curves were used to analyze survivorship and used the following end points: (1) death and (2) prosthesis revision for infection, radiographic loosening, or any other reasons with any components.

\section{Statistical analysis}

All continuous data were presented as mean \pm standard deviation (ranges). Two-sided, paired Student $t$ tests were utilized to analyze preoperative and last follow-up data in both groups. Two-sided, independent sample Student $t$ tests were utilized to analyze data between the two groups. All discontinuous data were presented as frequencies (percentages) and were analyzed by Pearson $\chi^{2}$ tests or Fisher's exact probability tests. The Wilcoxon rank-sum test was used to analyze ranked data. The Kaplan-Meier survival curves were compared with the Mantel-Cox log-rank test. All significance levels were set at $\alpha=0.05$, and $P<0.05$ indicated significant difference. All statistical analysis was calculated using SAS 9.4 (Statistical Analysis System, SAS Institute, Cary, NC, USA).

\section{Results}

\section{Clinical and functional results}

The duration of follow-up was $10.72 \pm 1.78$ years (range 10-12) in the SR group and was $10.65 \pm 1.87$ years (range 10-12) in the MR group. There were no significant differences in mean age, gender, BMI, or diagnoses of disease, as shown in Table 1.

All functional results of the two groups are listed in Table 2. No preoperative results differed between the two groups. Both groups showed significant improvements from preoperative values to their follow-up in terms of HSS, KSS, WOMAC score, SF-12, and ROM 


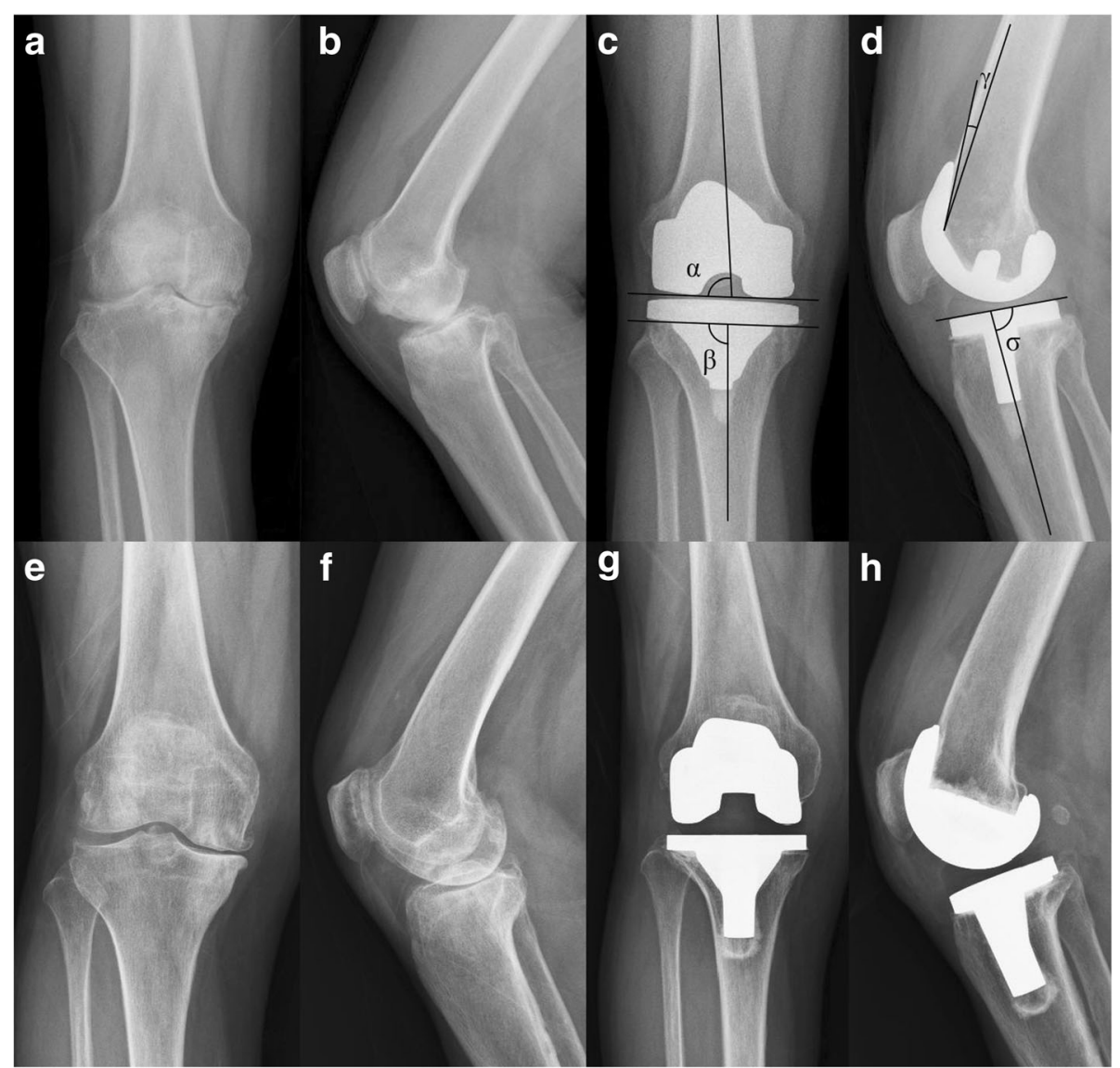

Fig. 2 a-d A 68-year-old female diagnosed with osteoarthritis underwent left knee arthroplasty with an SR (Scorpio NRG) prosthesis. a, b Preoperatively. c, d At 10-year follow-up. e-h A 65-year-old female diagnosed with osteoarthritis underwent right knee arthroplasty with an MR (PFC) prosthesis. e, f Preoperatively. $\mathbf{g}$, h At 10-year follow-up. All components were considered stable, and no radiolucent lines or osteolysis were detected. c, d Radiological evaluation angles

(flexion and extension). No significant differences were found between the groups. At the final follow-up, no significant differences were found in chair test results between the groups $(P=0.7799)$, as shown in Table 3 .

\section{Pain and crepitation}

The SR and MR groups showed no significant differences in VAS scores preoperatively $(P=0.5009)$. At the final follow-up, VAS scores decreased significantly in the two groups. There were no significant differences between the two groups. Nine (10.38\%) patients in SR reported AKP, and 17 (16.34\%) reported painless crepitation, significantly less than those in the MR group, with

Table 2 Summary of patients lost

\begin{tabular}{llll}
\hline Reason for drop out & SR group & MR group & Total \\
\hline Withdrew (not contactable) & 12 & 14 & 26 \\
Revised & 4 & 5 & 9 \\
Death & 3 & 4 & 7 \\
Total & 19 & 23 & 42 \\
\hline
\end{tabular}

20 (17.54\%) patients reporting AKP and 26 (28.07\%) reporting crepitation $(P<0.05)$. Severe isolated AKP had no significant differences at the final follow-up.

\section{Radiological results}

At the final 10-year follow-up, in 220 patients, radiolucent lines were found in 8 patients $(7.69 \%)$ in the SR group and in 10 patients (8.77\%) in the MR group, not significantly different $(P=0.1842)$, and all patients in both groups who had radiolucent lines were less than 2 $\mathrm{mm}$. No aseptic loosening of the tibial or femoral component, osteolysis, or infection were observed in either group (Fig. 2). At the final follow-up, angles measuring the component position showed no significant differences between the groups (Table 3).

\section{Satisfaction rate}

At the final follow-up, 76 (71.70\%) patients in the SR group were very satisfied and $23(21.69 \%)$ patients were satisfied. In the MR group, 74 (64.91\%) patients were very satisfied and $31(27.19 \%)$ patients were satisfied. 
Table 3 Results at final follow-up

\begin{tabular}{|c|c|c|c|}
\hline & SR $(n=106)$ & $\operatorname{MR}(n=114)$ & $P$ value \\
\hline \multicolumn{4}{|l|}{ Functional results } \\
\hline \multicolumn{4}{|l|}{ HSS } \\
\hline Preoperative & $41.23 \pm 5.76$ & $42.35 \pm 5.34$ & 0.1359 \\
\hline Final follow-up & $86.32 \pm 10.22$ & $84.52 \pm 10.53$ & 0.2002 \\
\hline \multicolumn{4}{|l|}{ KSS (knee) } \\
\hline Preoperative & $40.56 \pm 15.62$ & $42.63 \pm 17.36$ & 0.5700 \\
\hline Final follow-up & $84.52 \pm 18.50$ & $85.63 \pm 16.82$ & 0.6416 \\
\hline \multicolumn{4}{|l|}{ KSS (function) } \\
\hline Preoperative & $45.62 \pm 16.57$ & $44.58 \pm 17.56$ & 0.6517 \\
\hline Final follow-up & $86.23 \pm 17.50$ & $85.14 \pm 16.20$ & 0.6319 \\
\hline \multicolumn{4}{|l|}{ WOMAC score } \\
\hline Preoperative & $54.68 \pm 18.69$ & $56.23 \pm 17.52$ & 0.5261 \\
\hline Final follow-up & $21.83 \pm 15.60$ & $23.24 \pm 15.80$ & 0.5064 \\
\hline \multicolumn{4}{|l|}{ SF-12 } \\
\hline Preoperative & $28.84 \pm 6.45$ & $30.13 \pm 6.14$ & 0.1300 \\
\hline Final follow-up & $52.48 \pm 5.34$ & $51.21 \pm 5.35$ & 0.1394 \\
\hline \multicolumn{4}{|l|}{ ROM (flexion) } \\
\hline Preoperative & $105.52 \pm 10.78^{\circ}$ & $104.18 \pm 10.62^{\circ}$ & 0.3542 \\
\hline Final follow-up & $115.65 \pm 10.32^{\circ}$ & $115.50 \pm 10.13^{\circ}$ & 0.9135 \\
\hline \multicolumn{4}{|l|}{ ROM (extension) } \\
\hline Preoperative & $10.23 \pm 2.45^{\circ}$ & $10.34 \pm 2.23^{\circ}$ & 0.7277 \\
\hline Final follow-up & $3.42 \pm 1.22^{\circ}$ & $3.28 \pm 1.25^{\circ}$ & 0.4020 \\
\hline Chair test (complete) & $88(83.02 \%)$ & $93(81.58 \%)$ & 0.7799 \\
\hline \multicolumn{4}{|l|}{ Pain } \\
\hline \multicolumn{4}{|l|}{ VAS } \\
\hline Preoperative & $6.45 \pm 1.25$ & $6.57 \pm 1.38$ & 0.5009 \\
\hline Final follow-up & $0.42 \pm 0.15$ & $0.44 \pm 0.12$ & 0.2744 \\
\hline Anterior knee pain & $9(10.38 \%)$ & $20(17.54 \%)$ & $0.0251^{*}$ \\
\hline Painless crepitation & 17 (16.34\%) & $26(28.07 \%)$ & $0.0383^{*}$ \\
\hline Painful crepitation & $2(1.89 \%)$ & $3(2.63 \%)$ & 0.3287 \\
\hline \multicolumn{4}{|l|}{ Component evaluation } \\
\hline \multicolumn{4}{|l|}{ Radiological line } \\
\hline$<2 \mathrm{~mm}$ & $8(7.69 \%)$ & $10(8.77 \%)$ & 0.1842 \\
\hline$>2 \mathrm{~mm}$ & $0(0 \%)$ & $0(0 \%)$ & - \\
\hline $\operatorname{LDFA}(a)^{\circ}$ & $84.32 \pm 3.15$ & $84.57 \pm 3.25$ & 0.5635 \\
\hline $\operatorname{MDTA}(\beta)^{\circ}$ & $89.87 \pm 3.24$ & $89.56 \pm 3.32$ & 0.4846 \\
\hline $\operatorname{FEFA}(\gamma)^{\circ}$ & $6.54 \pm 1.68$ & $6.76 \pm 2.26$ & 0.4162 \\
\hline $\operatorname{TSA}(\sigma)^{\circ}$ & $88.25 \pm 3.53$ & $87.82 \pm 3.72$ & 0.3809 \\
\hline
\end{tabular}

*Continues data presented as the mean \pm std, Student's $t$ test was utilized. Discontinuous data presented as frequencies (percentages); Pearson's chisquared test or Fisher's exact test was utilized. $P<0.05$ indicates significant differences

Rank data in cross-tabulation (Table 4) with Wilcoxon rank-sum test showed no significant differences between the two groups $(Z=0.2091, P=0.4172)$.

\section{Complication and survivorship}

During the follow-up period, 12 patients withdrew in the SR group; 3 patients died at 5, 6, and 8 years, respectively; 1 patient underwent revision for periprosthetic fracture at 5 years; 2 patients underwent revision for prosthesis loosening at 7 years; and 1 patent underwent revision for prosthesis loosening at 8 years. Fourteen patients withdrew in the MR group; 4 patients died at 6, 7 , 8 , and 8 years, respectively; 1 patient underwent revision for periprosthetic fracture at 4 years; 2 patients underwent revision for prosthesis loosening at 6 years; 1 patient underwent revision for prosthesis loosening at 8 years; and 1 patient underwent revision for periprosthetic fracture at 9 years, as shown in Table 2. Kaplan-Meier survival estimated at 10 years was $94.40 \%$ (95\% CI 90.4-98.4\%) in the SR group and was $93.43 \%$ (95\% CI 89.4-97.4\%) in the MR group. There were no significant differences between the two groups by the Mantel-Cox log-rank test $\left(\chi^{2}=0.09997, P=0.7519\right.$, Fig. 3).

\section{Discussion}

We compared two types of posterior-stabilized prostheses: SR (Scorpio NRG, Stryker Orthopedics, Mahwah, NJ, USA) and MR (PFC, DePuy Orthopedics, IN, USA). We hypothesized that both the two prostheses can provide satisfactory results and the SR prostheses provide similar functional, radiological, and survival results to the MR prostheses. We discovered that the SR prostheses gave less anterior knee pain than did the MR prostheses, while other clinical and functional outcomes did not show any significant differences. Radiological results, complications, and survival of the prostheses were also similar.

We overviewed recent literature of the comparison between SR and MR prostheses and compared our current study to them. As is shown in Table 5, many of the previous studies had conflicting outcomes, none of them provided longer than 5 years follow-up [15-18, 22]. Although our study was a retrospective cohort study, it was the longest study which included at least 10-year follow-up and examined clinical, functional, radiological, and survival outcomes.

Table 4 Satisfaction rate at final follow-up

\begin{tabular}{llll}
\hline & SR $(n=106)$ & MR $(n=114)$ & Total \\
\hline Very satisfied & 76 & 74 & 150 \\
Satisfied & 23 & 31 & 54 \\
Uncertain & 5 & 7 & 12 \\
Unsatisfied & 2 & 2 & 4 \\
Total & 106 & 114 & 220 \\
\hline
\end{tabular}

Rank data cross tabulation table; discontinuous rank data presented as frequencies; according to Wilcoxon rank-sum test, $Z=0.2091, P=0.4172$, which indicates no significant differences 


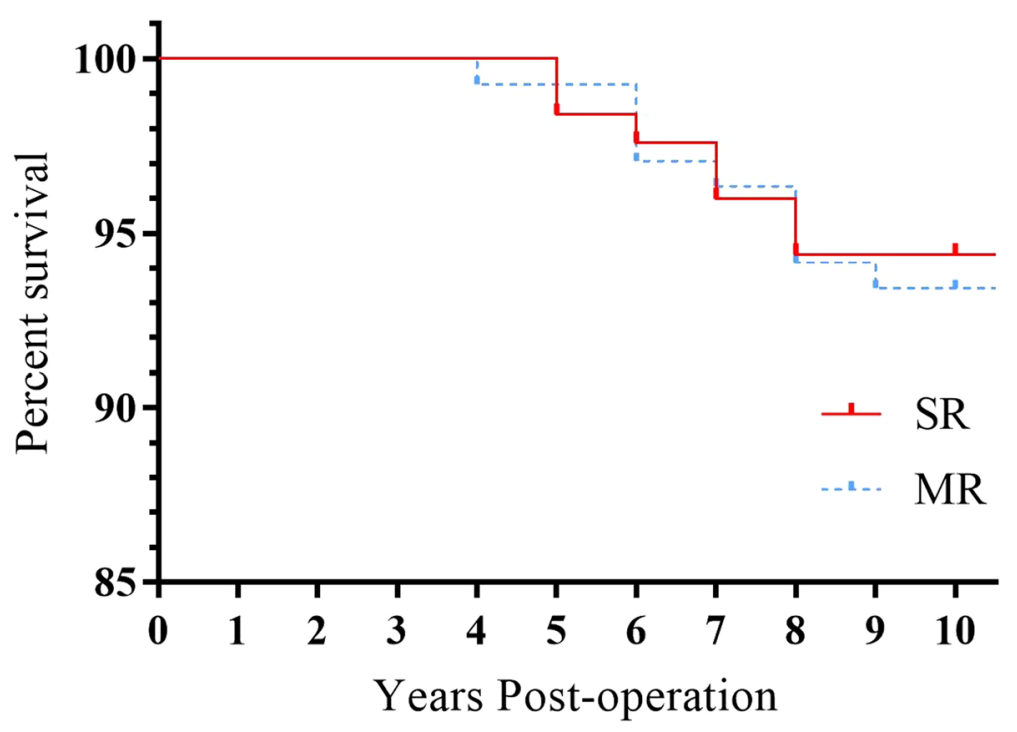

Fig. 3 Kaplan-Meier survival curve

Although TKA had wonderful results in treating severe osteoarthritis, nearly $25 \%$ of patients were dissatisfied after TKA, and up to $30 \%$ had anterior knee pain or painless noise [1]. MR prostheses were designed based on the J curve theory and have been the most widely utilized prostheses since the 1980s [6, 7]. SR prostheses were designed based on the theory that the actual FEA of the posterior femur condyles was considered a single-radius axis of a cylinder for flexion and extension of the knee through $10-120^{\circ}$. SR prostheses provide a more posterior flexion axis and an increased extensor moment arm to relieve the tension of the quadriceps. Additionally, SR designs maintain middle range stabilization and reduce the paradoxical anterior femoral movement to alleviate AKP.

Our study reported that the SR prostheses gave less anterior knee pain than did the MR prostheses. This is in line with Hamilton et al. [11], who compared 75 SR (Triathlon) with $90 \mathrm{MR}$ prostheses, reporting that the SR designs reduced the worst daily pain $(P=0.003)$ over 3 years of follow-up; however, Oxford Knee Scores $(P=0.09)$ and timed functional performance tasks $(P=0.23)$ did not reach statistical significance. Similarly, Palmer et al. [23] reported that $66.3 \%$ of patients with the SR prostheses experienced no pain, less than $54.4 \%$ with the MR prostheses. Anterior knee pain had related factors in some of the implant designs. Overstuffing of the knee joint, patella impingement, and instability of the knee may result to pain [24]. Similar sizes of the femoral implants were utilized in both groups, and patellar resurfacing was not utilized in neither patient in our study. Theoretically, SR provided a lateral flexion axis so the crepitus between patella and femoral implants may be less than MR. SR increased extensor moment arm to relieve the tension of the quadriceps as to led to better quadriceps efficiency. In the MR femoral implants, the length of the ligament changes at mid-flexion as the momentary axis changes from a long one to a short one, which may cause in instability, while the SR femoral design has a fixed main axis which maintains the tension of the collateral ligaments during the movement. All of these may explain our follow-up discovery.

SR had theoretical advantages that were likely to translate into satisfactory improved clinical outcomes. We did not observe significant improvement in terms of other clinical, radiological, and survival results when comparing the SR with the MR prostheses in TKA. Several possible reasons could explain this.

First, the clinical scales traditionally used to generate orthopedic results such as HSS and KSS score may not be sensitive enough to illuminate differences in prostheses designs [25]. Mahoney et al. [10] compared 83 patients with SR (Scorpio) to 101 patients with MR prostheses and reported that significantly more patients in the SR group were able to rise from a 16-in. chair without using their arms starting at 6 weeks and the difference was maintained through 2 years; however, there were no differences in the KSS scores between the two groups at 2 years. Jo et al. [14] compared 50 patients with SR (Scorpio NRG) and 50 patients with MR, using a navigation system to measure stability during $0^{\circ}, 30^{\circ}$, $60^{\circ}$, and $90^{\circ}$ of flexion, and reported that the SR group had better intraoperative stability, especially improved midrange stability from 60 to $90^{\circ}$, but no significant differences in the HSS scores or WOMAC scores. Larsen et al. [16] compared 16 patients with SR (Scorpio) to 16 patients with MR prostheses, using computer navigation for gait analysis, and reported that the SR implant had better kinematic properties at 1-year follow-up than 


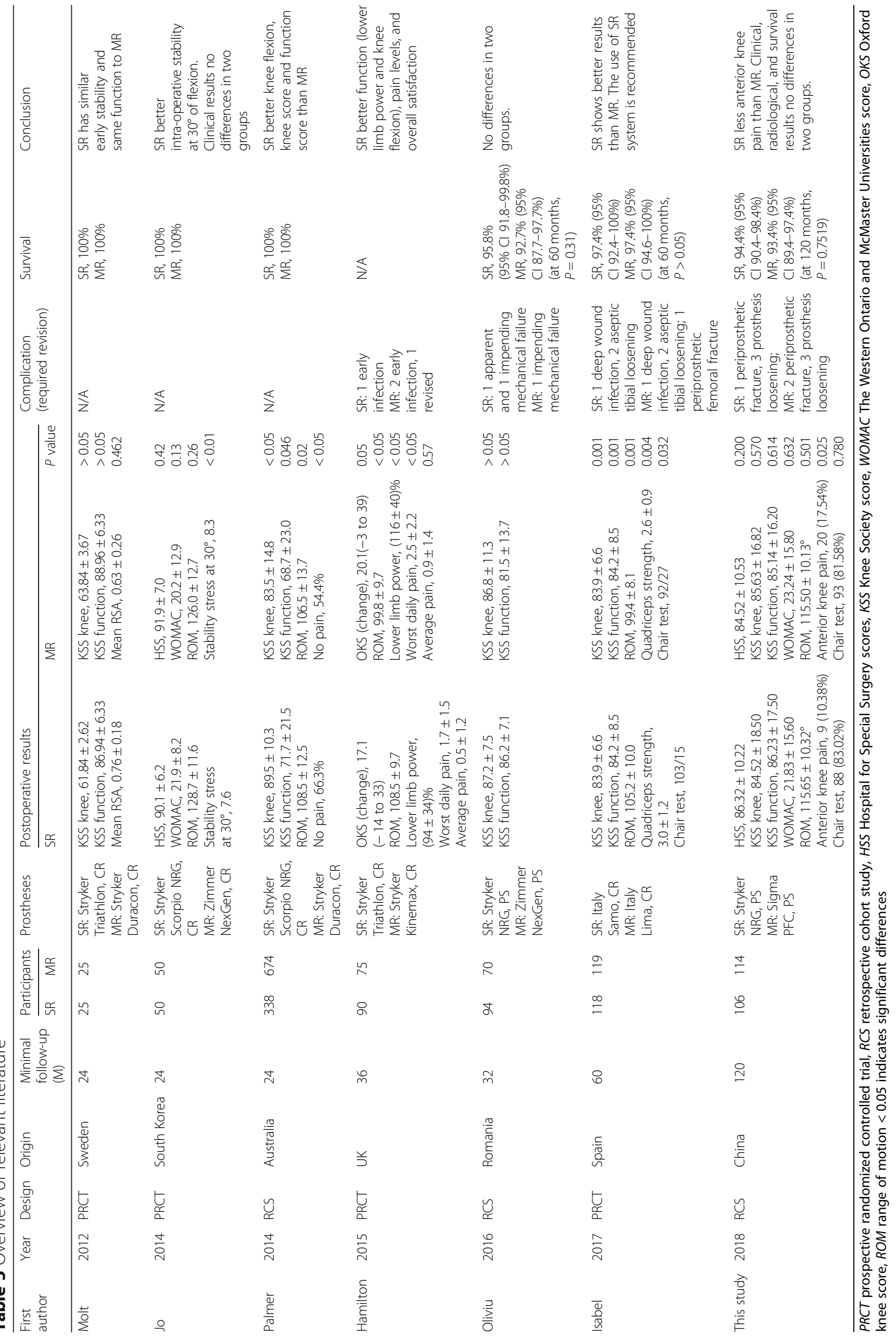


did the MR prostheses. MR prostheses remained more extended and had decreased power absorption during weight acceptance than did the SR prostheses. However, both surgical groups had similar KSS for knee and functional scores at the 1-year follow-up. Therefore, any advantages of the SR prostheses in terms of muscular recovery, component stability, or gait cycle may not be accurately reflected in the conventional function scales.

Second, soft tissue and gap balance are influenced by many factors, including different types of osteotomies and the effect of releasing the tight medial-lateral structures. Therefore, the stability of the knee could not be completely guaranteed by the SR prosthesis. Stoddard et al. [15] compared eight patients with SR (Triathlon) to eight patients with MR prostheses using a computer navigation system to measure the stability and reported that significant differences were not found between the types of femur design and that mid-range instability may have been related to unrecognized ligament laxity. Therefore, considering the complexity and multi-step process of the soft tissue balance, SR prostheses did not guarantee the stability of the knee at all angles.

Our study had several limitations. First, it was a retrospective cohort study such that selection bias would be expected. Nevertheless, our study reported long-term follow-up with a large population of consecutive patients. Second, more accurate tests are required to increase the sensitivity of the clinical and functional results.

\section{Conclusion}

Both SR and MR posterior-stabilized prostheses can lead to satisfactory outcomes. The SR prosthesis design gave less anterior knee pain than did the MR prostheses. The two prostheses showed no differences in terms of clinical scales, radiological results, satisfaction rate, or survival results at long-term follow-up, requiring more accurate measurements.

\footnotetext{
Abbreviations

AKP: Anterior knee pain; CR: Cruciate-retaining; FEFA: Flexion/extension femoral angle; HSS: Hospital for Special Surgery; KA: Kinematic alignment; LDFA: Lateral distal femoral angle; MA: Mechanical alignment; MCL: Medial collateral ligament; MDTA: Medial proximal tibia angle; MR: Multi-radius; PCL: Posterior cruciate ligaments; ROM: Range of motion; SF-12 Scale: Short Form-12 health survey; SR: Single-radius; TEA: Trans-epicondylar axis; TKA: Total knee arthroplasty; TSA: Tibial slope angle; VAS: Visual analog scale
}

\section{Acknowledgements}

We thank the nursing staffs from the Department of Orthopedic Surgery, West China Hospital, for the support and all the patients enrolled in the study. We thank American Journal Experts for the language assistance.

\section{Funding}

No external funding was received for this study.

\section{Availability of data and materials}

The datasets used and/or analyzed during the current study are available from the corresponding author on reasonable request.

\section{Authors' contributions}

ZL performed the data collection and analysis and manuscript writing. QX, $H W$, and FXP performed the data analysis. HW, ZYL, and ZKZ helped to draft the manuscript. All authors reviewed the final manuscript. All authors agree to be accountable for all aspects of the work. All authors read and approved the final manuscript.

\section{Ethics approval and consent to participate}

The retrospective cohort study was approved by the Institutional Review Board of West China Hospital, Sichuan University (ID number: 2012-268). The work was registered in the Chinese Clinical Trial Registry, (ID number: ChiCTR1800016129). Informed consent was obtained from all patients and their relatives. Information about deceased participants was collected from their relatives. All experiments were performed in accordance with the relevant guidelines and regulations. This study was conducted in accordance with the Declaration of Helsinki.

\section{Consent for publication}

Not applicable

\section{Competing interests}

The authors declare that they have no competing interests.

\section{Publisher's Note}

Springer Nature remains neutral with regard to jurisdictional claims in published maps and institutional affiliations.

Received: 4 September 2018 Accepted: 3 May 2019

Published online: 16 May 2019

\section{References}

1. Shan L, Shan B, Suzuki A, Nouh F, Saxena A. Intermediate and long-term quality of life after total knee replacement: a systematic review and metaanalysis. J Bone Joint Surg Am. 2015;97(2):156-68. https://doi.org/10.2106/ jbjs.M.00372.

2. Bin Abd Razak HR, Tan CS, Chen YJ, Pang HN, Tay KJ, Chin PL, Chia SL, Lo NN, Yeo SJ. Age and preoperative Knee Society Score are significant predictors of outcomes among Asians following total knee arthroplasty. J Bone Joint Surg Am. 2016;98(9):735-41. https://doi.org/10.2106/jbjs.15.00280.

3. Scott CE, Howie CR, MacDonald D, Biant LC. Predicting dissatisfaction following total knee replacement: a prospective study of 1217 patients. J Bone Joint Surg (Br). 2010;92(9):1253-8. https://doi.org/10.1302/0301-620x. 92b9.24394.

4. Frankel VH, Burstein AH, Brooks DB. Biomechanics of internal derangement of the knee. Pathomechanics as determined by analysis of the instant centers of motion. J Bone Joint Surg Am. 1971;53(5):945-62.

5. Kennedy JC, Fowler PJ. Medial and anterior instability of the knee. An anatomical and clinical study using stress machines. J Bone Joint Surg Am. 1971;53(7):1257-70.

6. Churchill DL, Incavo SJ, Johnson CC, Beynnon BD. The transepicondylar axis approximates the optimal flexion axis of the knee. Clin Orthop Relat Res. 1998;356:111-8.

7. Ranawat CS, White PB, West S, Ranawat AS. Clinical and radiographic results of attune and PFC sigma knee designs at 2-year follow-up: a prospective matched-pair analysis. J Arthroplast. 2017;32(2):431-6. https://doi.org/10. 1016/j.arth.2016.07.021.

8. Hollister AM, Jatana S, Singh AK, Sullivan WW, Lupichuk AG. The axes of rotation of the knee. Clin Orthop Relat Res. 1993;290:259-68.

9. Eckhoff DG, Bach JM, Spitzer VM, Reinig KD, Bagur MM, Baldini TH, Flannery NM. Three-dimensional mechanics, kinematics, and morphology of the knee viewed in virtual reality. J Bone Joint Surg Am. 2005;87(Suppl 2):71-80. https://doi.org/10.2106/jbjs.E.00440.

10. Mahoney OM, McClung CD, dela Rosa MA, Schmalzried TP. The effect of total knee arthroplasty design on extensor mechanism function. J Arthroplast. 2002;17(4):416-21.

11. Hamilton DF, Burnett R, Patton JT, Howie CR, Moran M, Simpson AH, Gaston P. Implant design influences patient outcome after total knee arthroplasty: a prospective double-blind randomised controlled trial. Bone Joint J. 2015;97B(1):64-70. https://doi.org/10.1302/0301-620X.97B1.34254.

12. Menciere ML, Epinette JA, Gabrion A, Arnalsteen D, Mertl P. Does high flexion after total knee replacement really improve our patients' quality of life at a short-term follow-up? : a comparative case-control study with 
hyperflex PFC Sigma versus a Triathlon knee series. Int Orthop. 2014;38(10): 2079-86. https://doi.org/10.1007/s00264-014-2372-4.

13. D'Lima DD, Poole C, Chadha H, Hermida JC, Mahar A, Colwell CW, Jr. Quadriceps moment arm and quadriceps forces after total knee arthroplasty. Clin Orthop Relat Res. 2001;392:213-20.

14. Jo AR, Song EK, Lee KB, Seo HY, Kim SK, Seon JK. A comparison of stability and clinical outcomes in single-radius versus multi-radius femoral design for total knee arthroplasty. J Arthroplast. 2014;29(12):2402-6. https://doi.org/10. 1016/j.arth.2014.03.033.

15. Stoddard JE, Deehan DJ, Bull AM, McCaskie AW, Amis AA. The kinematics and stability of single-radius versus multi-radius femoral components related to mid-range instability after TKA. J Orthop Res. 2013;31(1):53-8. https://doi.org/10.1002/jor.22170.

16. Larsen B, Jacofsky MC, Jacofsky DJ. Quantitative, comparative assessment of gait between single-radius and multi-radius total knee arthroplasty designs. J Arthroplast. 2015;30(6):1062-7. https://doi.org/10.1016/j.arth.2015.01.014.

17. Oliviu RC, Zazgyva A, Septimiu S, Ors N, Sorin PT. Mid-term results of total knee replacement with single-radius versus multi-radius posterior-stabilized implants. Acta Orthop Traumatol Turc. 2016;50(2):125-31. https://doi.org/10. 3944/aott.2015.15.0050

18. Wellman SS, Klement MR, Queen RM. Performance comparison of singleradius versus multiple-curve femoral component in total knee arthroplasty: a prospective, randomized study using the Lower Quarter Y-Balance Test. Orthopedics. 2017;40(6):e1074-80. https://doi.org/10.3928/0147744720171020-02.

19. Parratte S, Pagnano MW, Trousdale RT, Berry DJ. Effect of postoperative mechanical axis alignment on the fifteen-year survival of modern, cemented total knee replacements. J Bone Joint Surg Am. 2010;92(12): 2143-9. https://doi.org/10.2106/jbjs.l.01398.

20. Ewald FC. The Knee Society total knee arthroplasty roentgenographic evaluation and scoring system. Clin Orthop Relat Res. 1989;248:9-12.

21. Sarmah SS, Patel S, Hossain FS, Haddad FS. The radiological assessment of total and unicompartmental knee replacements. J Bone Joint Surg (Br). 2012;94(10):1321-9. https://doi.org/10.1302/0301-620X.94B10.29411.

22. Jenny JY, Miehlke R, Saragaglia D, Geyer R, Mercier N, Schoenahl JY, Thiel B. Single-radius, multidirectional total knee replacement. Knee Surg Sports Traumatol Arthrosc. 2013;21(12):2764-9. https://doi.org/10. 1007/s00167-012-2178-0

23. Palmer J, Sloan K, Clark G. Functional outcomes comparing Triathlon versus Duracon total knee arthroplasty: does the Triathlon outperform its predecessor? Int Orthop. 2014;38(7):1375-8. https://doi.org/10.1007/ s00264-014-2307-0.

24. Peralta-Molero JV, Gladnick BP, Lee YY, Ferrer AV, Lyman S, Gonzalez Della Valle A. Patellofemoral crepitation and clunk following modern, fixedbearing total knee arthroplasty. J Arthroplast. 2014;29(3):535-40. https://doi. org/10.1016/j.arth.2013.08.008.

25. Gomez-Barrena E, Fernandez-Garcia C, Fernandez-Bravo A, Cutillas-Ruiz R, Bermejo-Fernandez G. Functional performance with a single-radius femoral design total knee arthroplasty. Clin Orthop Relat Res. 2010;468(5):1214-20. https://doi.org/10.1007/s11999-009-1190-2

Ready to submit your research? Choose BMC and benefit from:

- fast, convenient online submission

- thorough peer review by experienced researchers in your field

- rapid publication on acceptance

- support for research data, including large and complex data types

- gold Open Access which fosters wider collaboration and increased citations

- maximum visibility for your research: over $100 \mathrm{M}$ website views per year

At BMC, research is always in progress.

Learn more biomedcentral.com/submissions 